\title{
(Un)doing gender empirisch: Konzeptionelle, methodische und praktische Schlussfolgerungen
}

\author{
Julia C. Nentwich und Franziska Vogt
}

\section{1 (Un)doing gender untersuchen: Eine Methodologie des Explizierens, Reflektierens und Kontrastierens}

In unserem Forschungsprojekt ,,(Un)doing gender in der Kita“ haben wir uns vorgenommen, möglichst vielfältige theoretische Konzeptionen von doing und undoing gender empirisch zu untersuchen. Die eingangs näher ausgeführten konzeptionellen Überlegungen von Deutsch und Hirschauer zu undoing gender, von Goffman und Faulstich-Wieland zur Dramatisierung und Entdramatisierung, von Butler zur Performativität und Subversion sowie den diskurspsychologischen Konzepten der Subjektpositionierung, bis hin zu den zeitlichen, materiellen und körperlichen Praktiken bei Connell, Gherardi und Martin und in Verbindung mit Überlegungen wie denen Ackers zur gendered organisation oder den professionstheoretischen Reflexionen Rabe-Klebergs boten uns Hand zum Experimentieren mit und Erproben von verschiedenen konzeptionellen und methodischen Zugängen, Möglichkeiten und Herausforderungen.

Doing und undoing gender muss als episodenhaft hergestellt untersucht werden, so das Fazit der die ethnomethodologische Omnirelevanzannahme problematisierenden Diskussionen. Gender steht zwar in allen Interaktionen als möglicher Interpretationsrahmen zur Verfügung und ist damit omnipräsent, muss jedoch nicht zwingend aktiviert werden. Die Möglichkeit eines undoing gender

\author{
J. C. Nentwich ( $\varangle)$ \\ Universität St. Gallen, St. Gallen, Schweiz \\ E-Mail: julia.nentwich@unisg.ch \\ F. Vogt \\ Pädagogische Hochschule St. Gallen, St. Gallen, Schweiz \\ E-Mail: franziska.vogt@phsg.ch
}


war somit eingeführt und brachte zugleich die Herausforderung mit sich, wie empirisch entschieden werden kann, wann in einer Situation gender gemacht, wann infrage gestellt und wann ruhen gelassen wird. Ein episodenhaftes Verständnis von (un)doing gender erfordert eine Operationalisierung von doing wie auch von undoing gender (Nentwich und Kelan 2014). Diese ist jedoch zugleich mit der Gefahr verbunden, Geschlecht in die Analyse einer Situation einzubringen und so der Fluidität von gender nicht gänzlich Rechnung tragen zu können. Kurz, es besteht die Gefahr, Geschlecht zu reifizieren. Insbesondere in einem Kontext wie der Kita stellt dies eine besondere Herausforderung für die empirische Forschung dar, da Geschlechtsidentität nur ein Thema unter vielen darstellt, das hier im Alltag verhandelt wird. Wie aber können wir entscheiden, dass es Geschlecht ist, das in einer Situation zum Thema gemacht wird, während es in einer anderen ruhen gelassen wird, um dann in einer nächsten wieder dramatisiert zu werden? Das von Schegloff (1997) vorgebrachte Kriterium, gender müsse in der interpretierten Situation von den hieran beteiligten Akteuren auch relevant gemacht werden, um interpretierbar zu sein, würde den performativen Charakter von gender als sozialer Kategorie vernachlässigen. Wie von Wetherell (1998) gefordert, braucht es eine sozialtheoretische Fundierung des Zusammenhangs zwischen dem, was situativ getan wird und den zur Interpretation herangezogenen institutionalisierten Wissensbeständen. Goffmanns (1994/1977) Begriff der institutionellen Reflexivität, die diskurstheoretische Fundierung von Positionierungspraktiken (Nentwich und Morison 2018; Wetherell und Edley 2014), das von Gherardi (1994, 1995), Bruni et al. (2005) sowie Martin (2003, 2004) näher ausgeführte praxistheoretische Verständnis, wie auch Connells (2005) Theorie der hegemonialen Männlichkeit haben uns hierfür die notwendige sozialtheoretische Basis geboten. Die verschiedenen Ansätze verbindet ein Verständnis von gender als situativ und performativ hervorgebrachter Wirklichkeit, die jedoch nie ausschließlich situativ interpretiert werden kann (Wetherell 1998). Eine situative Inszenierung greift immer auf bereits etablierte, institutionalisierte Wissensbestände zurück. Um das situative gendering $\mathrm{zu}$ verstehen, muss darum zwingend Wissen über die in diesem Kontext zur Anwendung kommenden gendered practices erlangt werden (Martin 2003).

Als Forschende müssen wir in der Lage sein, unsere konzeptionellen Verständnisse von Geschlecht bzw. von doing und undoing zu explizieren. Nur so können sie kritisch reflektiert (Kelle 2016) und auch intersubjektiv nachvollziehbar gemacht werden. Für jede unserer empirisch untersuchten Forschungsfragen haben wir darum explizit und präzise geklärt, was jeweils unter doing und was unter undoing gender $\mathrm{zu}$ verstehen ist. Hierfür haben wir uns auf den Forschungsstand bezogen und darauf aufbauend, wie auch auf der Basis der eigenen Erhebungen das Verständnis von doing gender und undoing gender und 
not doing gender in Bezug auf die Interpretation der Daten und die Fragestellung operationalisiert. Über den Forschungsstand ließen sich zum Beispiel die für die Analyse von Raum und auch Interaktionen notwendigen Fragen nach der geschlechterdifferenzierenden Konnotation von Spielzeug und Spielangeboten klären. Die Stereotypenforschung sowie Statistiken zur Geschlechterdifferenzierung von Tätigkeiten und Berufen wie auch die historische Einbettung des Diskurses um den Raum als Bildungsakteur halfen hier, die vorgefundenen Genderismen zu bestimmen. Die historische Entwicklung der Kita als Einrichtung und die entsprechenden Diskurse für das Berufsbild schärften die für unsere Analyse von Subjektpositionen, Rhythmen und Routinen benötigten Bilder der Figur des guten Kinderbetreuers bzw. der guten Kinderbetreuerin. Das Verständnis von hegemonialer Männlichkeit, mit der die beobachteten Handlungen interpretiert werden können, erlaubte die Analyse der körperlichen Praktiken männlicher Kinderbetreuung. Die präzise Explizierung, was mit doing und undoing gender jeweils verstanden werden kann und welche Bedeutungskontexte den verschiedenen Verständnissen zugrunde liegen, dienten auch der Analyse der Ausbildungsmaterialien, die eine multiple und verdichtete Form der Konstruktion von Geschlechterwissen darstellen.

Da (un)doing gender nicht nur durch sprachliche, sondern auch durch schweigsame soziale Praktiken hergestellt wird (Hirschauer 2001), haben wir Artefakte und räumliche Arrangements, Praktiken und Routinen untersucht und die hierin eingeschriebenen Wissensbestände herausgearbeitet. Es handelt sich dabei um Wissensbestände, die für selbstverständlich gehalten werden und den Akteurinnen und Akteuren in der zu interpretierenden Sequenz nicht zwingend zugänglich sein müssen. Die Untersuchung von doing und undoing gender als episodenhaftem Geschehen in der Kita bringt so die Notwendigkeit mit, den relevanten Kontext, das jeweils verwendete Geschlechterwissen sowie die historischen Hintergründe für die Interpretation mit dem Ziel heranzuziehen, die Analyse mit den im jeweiligen Feld vorhandenen Wissensbeständen abstützen zu können.

Durch das im Forschungsdesign angelegte multimethodische und multiperspektivische Vorgehen konnten wir Beobachtungen jeweils mit Aussagen aus Interviews belegen oder aber auch herausfordern. Informationen über die Verwendung bestimmter Bedeutungen und Bezugnahmen im Interviewmaterial, deren Überprüfen und Hinterfragen in ethnografischen Beobachtungen und im Gespräch mit den Kitaleitungen und Kinderbetreuerinnen und -betreuern erscheinen uns zwingend, um über die Verwendung des jeweiligen Geschlechterwissens im untersuchten Feld Auskunft geben zu können. Wir verstehen das in den vielfältigen geführten Interviews stattfindende Reden über den Kita-Alltag nicht als reines Abbild desselben, sondern als ,eine spezifische Form von Praxis“ 
(Nentwich 2014, S. 57). Folglich sind die hierbei zu beobachtenden Subjektivierungspraktiken als Auseinandersetzung mit den in diesem spezifischen Kontext wirksamen Bedeutungen von Geschlecht, Weiblichkeit und Männlichkeit, aber auch Kinderbetreuung, Professionalität oder Führung zu analysieren und höchst aufschlussreich für die Wirkmächtigkeit bestimmter Diskurse und Anrufungen in diesem Kontext. Das ethnografische Vorgehen ermöglicht die Lesbarkeit des Gesagten in interpretierendem Kontrast zu dem, was im Kita-Alltag getan wird (Khan und Jerolmack 2013). Indem wir die verschiedenen methodischen wie auch analytischen Zugänge in diesem Forschungsprojekt immer wieder aufeinander bezogen diskutiert haben, konnten Interpretationen im Material begründet und die jeweilige Angemessenheit und Passung legitimiert werden.

Gerade diese Verschränkung von verschiedenen Daten und Analysezugängen machte es auch deutlich, dass die gendered practices im Feld der Kita oft impliziten Ordnungsstrukturen folgen. Allerdings findet auf der Mikroebene ein stetiger Wechsel zwischen doing und undoing statt, durch den unterschiedliche und widersprüchliche Wissensbestände zugleich wirkmächtig werden können. So zeigt die im achten Kapitel dargestellte Analyse des in den Ausbildungscurricula verwendeten Geschlechterwissens, dass doing und undoing gender nicht immer geordnet stattfinden, sondern in der Regel nebeneinander und unter Umständen auch auf widersprüchliche Weise. Soll der binäre Rahmen von doing als Differenz betonend und undoing als Gleichheit betonend (Deutsch 2007) verlassen werden, der immer ein Wissen über die binäre Unterscheidung voraussetzt und wiederum in die Interpretation einfließen lässt, erscheint uns ein episodenhaftes, durch schnelle, fluide Wechsel gekennzeichnetes Verständnis von (un)doing gender für die empirische Analyse zielführender. Dieses beinhaltet zugleich die Annahme einer situativ notwendigen Entscheidung über das jeweils vorliegende doing oder aber undoing. Mit einem Blick auf die Fluidität von (un)doing gender wird leichter sichtbar, dass das eine in das andere übergehen kann oder aber sogar beide Interpretationen in ein und derselben Situation zulässig wären.

Neben diesem Plädoyer für eine größtmögliche Offenheit hinsichtlich der empirisch zu erwartenden Wirkweisen von (un)doing gender müssen wir jedoch auch betonen, dass auch ein entlang stereotyper Wissensbestände definiertes Verständnis wertvolles Wissen über die Herstellungsweisen von Geschlecht liefert. So zeigt die Analyse der Interaktionen zwischen Kinderbetreuenden und Kindern, dass die interpretative Verwendung von stereotypen Wissensbeständen durchaus produktiv sein kann und nicht zwingend für alle Forschungsfragen aus der Befürchtung heraus vermieden werden sollte, die Verwendung von Geschlechterstereotypen als Analysekategorien würde zwangsläufig Geschlecht reifizieren. Nur indem wir klar definiert haben, was wir in einer Interaktion als doing und damit 
als typische Aktivität, und was als undoing, und damit als untypische Aktivität für das jeweilige Geschlecht für diese Analyse verstehen wollen, wurde es möglich, die Interaktionsverläufe über mehrere turns hinweg zu betrachten. Ähnliches zeigt die Analyse der körperlichen Praktiken männlicher Kinderbetreuer. Ohne die Verknüpfung von beobachteten Praktiken mit einem auf Connells Theorie hegemonialer Männlichkeit (2005) abgestützten Verständnisses davon, was als hegemonial männliches Handeln interpretiert werden kann, wären hier keinerlei Aussagen über Geschlecht in den beobachteten Situationen möglich. Auch ein Sprechen über Identität als männlicher Kinderbetreuer oder aber weibliche Kinderbetreuerin ist nur möglich, wenn Geschlecht explizit thematisiert und dadurch auch Differenz in der Interviewsituation dramatisiert wird. Gerade diese Dramatisierung ermöglicht es zu untersuchen, inwieweit bereits routinierte Umgangsweisen mit der für männliche Kinderbetreuer ausgelösten Infragestellung bestehen oder aber eine Irritation ob der Relevanz dieser Frage im Fall der weiblichen Kinderbetreuerin besteht. Differenz wurde hier durch die Forschungsfrage nach der spezifischen Position von Männern in der Kita zwar dramatisiert, die empirischen Ergebnisse zeigen jedoch, dass die Interviewpartner wiederum auf unterschiedliche Weise situativ mit dieser Dramatisierung umgehen konnten. Männer betonten nur in wenigen Fällen mit den durch sie eingebrachten Innovationen und Besonderheiten Differenz, vielmehr waren sie darum bemüht, als Gleiche angesehen zu werden und gender zu dethematisieren. Die kritische Diskussion dieser Reifikation von Differenz in der Frage nach der spezifischen Situation von Männern in der Kita durch das Forschungsteam ermöglichte, diese Intervention bei der Interpretation des Materials wiederum kritisch zu reflektieren. Diese Beispiele zeigen, dass die methodische Einführung eines klaren Konzepts von Differenz, bzw. Männlichkeit und Weiblichkeit, wie auch von Gleichheit, weiterführende Erkenntnisse über (un)doing gender erlaubt, jedoch sowohl methodisch als auch konzeptionell reflektiert werden muss (Kelle 1999, 2005).

Unsere Konzeption von doing und undoing gender als sowohl dramatisierbar, d. h. in den Mittelpunkt zu stellen, als auch dethematisierbar, d. h. im Hintergrund verbleibend, hat sich hier als besonders fruchtbar erwiesen. Durch die analytische Trennung dieser zwei Dimensionen in unserer Analyse der Interaktionen wie auch der Subjektpositionierungen wurde es möglich, den Inhalt (doing oder undoing) von der jeweiligen Modalität (dramatisiert oder dethematisiert) zu unterscheiden und so die Dynamik in den Interaktionen analysierbar zu machen. Indem (un)doing gender als fluide und episodenhaft untersucht wurde, konnten genau diese Verläufe und fortlaufenden Wechsel von Dethematisierung und Dramatisierung mikroanalytisch sichtbar gemacht werden. 
Eine Analyse von (un)doing gender in der Kita richtet den Blick weg vom Individuum hin auf die es konstruierenden Praktiken. Fragen wie „Machen Männer als Kinderbetreuer etwas anderes?“ oder „Spielen Mädchen immer nur mit Puppen, Jungen nur mit Autos?" können auf diese Weise neu beantwortet werden. Der Blick führt dabei über das Individuum hinaus und beleuchtet sowohl die institutionellen Arrangements des Andersmachens als auch deren Notwendigkeiten und Effekte. Wie auch bereits Brandes et al. (2016) in ihrer Tandem-Studie gezeigt haben, ist es weniger das Geschlecht der Fachpersonen, das hier eine Rolle spielt, als das Geschlecht des Kindes, mit dem sie pädagogisch interagieren. Geschlecht wurde im Kontext der von uns untersuchten Kitas von den männlichen Kinderbetreuern vor allem dann relevant, wenn es durch Kontrastierung mit dem anderen Geschlecht dramatisiert wurde. Hegemoniale Männlichkeit, ein doing gender der Kinderbetreuer wird insbesondere dann betont, wenn durch den entsprechenden situativen Kontext eine Dramatisierung notwendig erscheint. Undoing gender hingegen wird in Zusammenhang mit gleichen Rechten und Schutz vor Diskriminierung betont. Um diesen kontextspezifischen, fluiden Wechsel zwischen doing und undoing, Dramatisierung und Dethematisierung empirisch untersuchen zu können, braucht es mehrperspektivische und multimethodische Zugangsweisen.

\subsection{Multimethodische und mehrperspektivische Ethnografie}

Ethnografie umfasst per se verschiedene Zugänge wie auch einen dynamischen Wechsel zwischen Datenerhebung und -analyse. Im Forschungsprojekt wurde eine Struktur der fortlaufenden Fokussierung angelegt: 20 Kitas waren an der visuellen Ethnografie und den Interviews mit den Kitaleitungen beteiligt, aus diesen wurde in acht Kitas, die Kinderbetreuer beschäftigten, Interviews mit Kinderbetreuern und Kinderbetreuerinnen geführt. Wiederum daraus wurden nach dem Prinzip des contrasting sampling (Schreier 2010) vier Kitas für die Beobachtung und Videografierung ausgewählt. Das Sampling erfolgte strukturiert und systematisch. Es ist jedoch hervorzuheben, dass kein repräsentatives oder zufälliges Sample angestrebt wurde - da insgesamt nur wenige Kitas überhaupt Männer als Kinderbetreuer beschäftigen, war es entscheidend, solche Kitas anzusprechen und zur Teilnahme zu motivieren. Bei der Rekrutierung zeigte sich, dass das Thema gender in der Kita die Praxis stark bewegt: Das Interesse und die Bereitschaft, am Forschungsprojekt teilzunehmen, war groß. Obwohl die Stichprobe keineswegs repräsentativ 
sein kann, scheinen dennoch keine besonderen Stichprobeneffekte vorhanden zu sein.

Für das ethnografische Forschungsdesign erschien es uns entscheidend, dass verschiedene methodische Zugänge gewählt werden. Diese verschiedenen Zugänge wurden zur Triangulation (Flick 2010) herangezogen, besonders zwischen Handeln und Reden (Nentwich 2014) wurden immer wieder neue Bezüge und Brüche identifiziert. Ebenso zeigte sich, dass jeder der methodischen Zugänge die je eigene Analyse der Performanz und der Ordnungsstrukturen von doing und undoing gender im Kontext der Kita erlaubte. So stellte sich in der Analyse mittels visueller Ethnografie ein starkes doing gender im räumlichen Arrangement heraus, das in den verschiedenen Spielarten von doing und undoing gender sowie Dramatisierung und Dethematisierung in den Interaktionen zwischen Kinderbetreuenden und Kindern in der Mikroanalyse als fluide und prozesshaft identifiziert werden konnte, und das in den Subjektpositionierungen der männlichen Kinderbetreuer weiter einen fortwährenden Wechsel zwischen Positionen der Geschlechterdifferenz und -gleichheit aufwies.

Indem wir die verschiedenen methodischen wie auch analytischen Zugänge in diesem Forschungsprojekt immer wieder aufeinander bezogen diskutiert haben, konnten Interpretationen im Material begründet und die jeweilige Angemessenheit und Passung legitimiert werden. Einschränkend muss hervorgehoben werden, dass das Sampling zwar systematisch erfolgte, jedoch keineswegs Repräsentativität beanspruchen kann. Durch das kriterienbasierte Vorgehen des theoretical sampling wurde sichergestellt, dass die Vielfalt der Kitas in der Auswahl reflektiert wurde. Die ausgewählten Kitas repräsentieren damit für die Deutschschweiz typische Kitas, in den zahlreichen Transferveranstaltungen fanden die Befunde sehr viel Resonanz, was darauf hindeutet, dass sie für die Deutschschweizer Kitas Geltung haben. Als weitere Einschränkung ist zu bemerken, dass die Dauer der ethnografischen Beobachtung in den vier Kitas sehr beschränkt war. Mehr Kitas oder längere Beobachtungen könnten die hier herausgearbeiteten Aspekte ergänzen, vertiefen und infrage stellen. Weiter ist einschränkend zu erwähnen, dass das ethnografische Forschungsdesign hier wenig Gelegenheit bot, die Erkenntnisse mit den Forschungsteilnehmenden zu diskutieren und im Sinn einer respondent validation zu reflektieren (Jeffrey und Troman 2004). Hingegen wurden die Erkenntnisse des Forschungsprojekts breit und vielfältig in zahlreichen Transferveranstaltungen wie auch im Entwicklungsprojekt „Gender in der Kita: Veränderungen zur Inklusion von Männern gemeinsam gestalten“ vertieft mit der Praxis diskutiert.

Gerade weil doing und undoing gender als performativ und fluide zu verstehen ist, ist die Analyse der ausgewählten Kitas, theoriegeleitet aufgrund von strukturellen Elementen ausgewählt, eine Herausforderung. Das Forschungsteam 
diskutierte die Daten ausgiebig und vertieft, innerhalb des Forschungsteams wurde in Bezug auf die hier dargestellten Ergebnisse Intersubjektivität hergestellt. Die Analyse der erhobenen Daten erforderte eine Fokussierung: So wurden bestimmte Videoausschnitte ausgewählt, kategorisiert und danach interpretiert, und es wurden Stellen aus den Ausbildungscurricula für eine detaillierte Analyse ausgewählt, ebenso sind die Fotografien einzelner Details aus den räumlichen Arrangements von der Auswahl der Forschenden geprägt. Die vorgenommene Auswahl wurde jedoch immer diskutiert. Die zahlreichen und intensiven Veranstaltungen, die das Forschungsteam in den Transferprojekten wie auch in weiteren Aktivitäten der Dissemination realisierte, ließen aus den Diskussionen die Schlüsse zu, dass auch die Akteurinnen und Akteure im Feld der Kita unsere Beobachtungen, Analysen und Schlussfolgerungen nachvollziehen können.

Die vertiefende Analyse innerhalb der methodischen Zugänge wie auch die fortlaufende, vergleichende Zusammenführung erwiesen sich als wesentlich für die Analyse von (un)doing gender wie auch zur Herausarbeitung von Handlungsmöglichkeiten für die Praxis. Das Forschungsprojekt war als Grundlagenforschungsprojekt angelegt. Aufgrund der empirischen Erkenntnisse zeigte sich jedoch bald das Potenzial des Transfers in die Praxis. Die zahlreichen Diskussionen mit Praktikerinnen und Praktikern aus den Kitas erwiesen sich als äußert fruchtbar und flossen ebenfalls in dieses Buch ein. Anregungen für die Praxis wurden weiter an vielen Veranstaltungen diskutiert und in zwei Praxisleitfäden formuliert (Tennhoff et al. 2014, 2017).

\subsection{Implikationen für die Praxis}

Mit unserem detaillierten und vertieften Blick auf (un)doing gender in der Kita haben wir aufgezeigt, dass das individuelle Handeln von Männern und Frauen stark vom jeweiligen Kontext geprägt ist. Männer positionieren sich als Mann und als Kinderbetreuer und sind darum bemüht, die beiden Positionierungen miteinander zu vereinbaren, um ein Erleben von Identitätsdissonanz zu verhindern. Das ideale Bild der Kinderbetreuung ist weniger von individuellen Idealvorstellungen und damit verknüpften Selbstbildern abhängig, als von den in der Kita etablierten Rhythmen und Routinen und den damit verankerten Aspekten von Bildung und/oder Betreuung. Doing masculinity erhält je nach Kontext eine unterschiedliche Wichtigkeit und auch das in den Interaktionen zwischen Betreuenden und Kindern gezeigte doing oder undoing gender scheint stark von den in der Organisationskultur verankerten Werten und Zielen bestimmt zu sein. 
Gender in der Kita zu verändern muss, so unsere Forschungsergebnisse, zwingend über eine Veränderung individueller Einstellungen und Verhaltensweisen hinausgehen. Es sind weniger die Männer in der Kita, die gender in die Kita bringen, als die Organisationskultur der Kita, die undoing gender und Dethematisierung mehr oder weniger fördert. Hierin besteht die unmittelbare Relevanz unserer Forschungsergebnisse für die Alltagspraxis der Kindertagesstätten. (Un)doing gender ist in die Rhythmen, Routinen, Räume und Interaktionen eingeschrieben. Da es sich um soziale Alltagspraktiken handelt, sind diese jedoch auf äußerst subtile Weise stabil verankert und nur schwer einer Reflexion zugänglich, die jedoch für die notwendigen Veränderungsprozesse wichtig wäre. In den zwei aus diesem Forschungsprojekt entstandenen Transferprojekten ${ }^{1}$ haben wir diese Feststellung zum Anlass genommen, zum einen durch Weiterbildungen von Kitaleitungspersonen, zum anderen in der Zusammenarbeit mit verschiedenen Kitateams in Form von Organisationsentwicklungen, diese subtilen gendered practices der Reflexion zugänglich zu machen und gezielt Veränderungen im organisationalen Alltag zu initiieren (Tennhoff et al. 2014, 2017). Unsere Befunde zum doing und undoing gender in den Interaktionen zwischen Kindern und Betreuenden wie auch zu den räumlichen Arrangements lassen zudem Schlussfolgerungen hinsichtlich einer geschlechtergerechten Pädagogik zu.

\subsubsection{Organisationsentwicklung in der Kita: Geschlechtergerechtigkeit als Ziel}

Für die Initiation organisationaler Veränderungsprozesse ist wesentlich, auch die impliziten, nichtsprachlich verfügbaren Verständnisse der jeweils geltenden Organisationskultur thematisierbar zu machen. Ein Zugang über die in der Organisation verwendete Symbolik und insbesondere Artefakte scheint dafür zielführend, da die Artefakte zunächst als konkret vorhandene und räumlich angeordnete Gegenstände wahrgenommen, und in ihrem Aufforderungscharakter im Hinblick auf

\footnotetext{
${ }^{1}$ Im Rahmen des durch den Schweizerischen Nationalfonds geförderten Wissenstransfers als Teil des des Nationalen Forschungsschwerpunkts „Gleichstellung der Geschlechter“ wurden im Jahr 2014 Workshops mit Kitaleitungen durchgeführt. Von Juni 2014 bis Mai 2017 führten wir das Projekt „Gender in der Kita: Veränderungen zur Inklusion von Männern gemeinsam gestalten" durch. Wir initiierten und begleiteten Organisationsentwicklungsprozesse in verschiedenen Kitas und boten eine Weiterbildungsgruppe für männliche Kinderbetreuung an. Dieses Projekt wurde durch das Eidgenössische Büro für die Gleichstellung von Frau und Mann im Rahmen der Finanzhilfen nach dem Gleichstellungsgesetz unterstützt.
} 
gender reflektiert werden können. Für eine Kitaentwicklung mit dem Auftrag, Geschlechtergerechtigkeit zu entwickeln, ist darum zentral, einerseits den involvierten Fachpersonen ein Wissen über Geschlecht als in den alltäglichen Interaktionen, Diskursen, Artefakten, Rhythmen und Routinen hergestellt zu vermitteln, und andererseits das analytische Werkzeug zur Verfügung zu stellen, das die entsprechenden Perspektiven für eine Betrachtung der eigenen Organisation eröffnet.

Organisationsentwicklungsprozesse benötigen zwar das gesamte Team, müssen aber in jedem Fall von der Leitung unterstützt werden. In den aus dem Forschungsprojekt heraus entwickelten Praxisbroschüren (Tennhoff et al. 2014, 2017) sowie in den darauf aufbauenden Weiterbildungsveranstaltungen und begleitenden Coachings fokussierten wir darum explizit auf die Leitungspersonen. Gender zum Thema zu machen wird hier zur Führungsaufgabe. Entlang der drei Themenbereiche „Frauen und Männer im Team“, „Raum und Angebot“ sowie „Zeiten, Ordnungen und Strukturen" vermittelten wir die im Forschungsprojekt entwickelten Erkenntnisse und richteten das Augenmerk der Leitungspersonen auf die häufig für selbstverständlich gehaltenen Abläufe und Aufgaben (Tennhoff et al. 2014). Die Broschüre soll ermöglichen, die Perspektive von gender im Kita-Alltag anzuwenden und Reflexion möglich zu machen, da die „Auswahl, Führung und Anleitung von Kinderbetreuerinnen und -betreuern im gemischten Team, die Entwicklung von in der Kita geltenden Regeln und Normen, wie auch die Gestaltung der räumlichen Situation und grundlegenden Arbeitsabläufe“ Leitungsaufgaben sind, ,die das Potential haben, den Kita-Alltag für Mitarbeiterinnen, Mitarbeiter und Kinder geschlechtergerechter zu gestalten“ (Tennhoff et al. 2014, S. 1). Durch die reduzierte und konkrete Darstellung der Forschungsbefunde und die darauf aufbauenden Tipps und Impulse für die Kitaleitungen wurde eine handlungsorientierte Form des Wissenstransfers erreicht. Ziel ist, gender aus der diffusen Omnipräsenz herauszuholen und durch die Reflexion von vielschichtigen, alltäglichen Praktiken auf der Mikroebene der Teamarbeit und Arbeitsaufteilung, der Interaktionen und körperlichen Praktiken wie auch der Raumgestaltung und ihrer Veränderung, die Organisationskultur der Kita so zu verändern, dass die Gleichstellung der Geschlechter vermehrt stattfinden kann.

\subsubsection{Inklusion von Männern}

Männer in der Kita müssen vielfältigen Erwartungen in Bezug auf gender gerecht werden, indem sie zugleich Männer repräsentieren sollen und dem weiblich 
konnotierten Ideal der Kinderbetreuungsperson entsprechen sollen. So beschreiben die Kinderbetreuer, dass ihnen eine Art Ersatzvaterrolle bei Kindern von alleinerziehenden Müttern zugewiesen wurde, und sie berichten davon, welche Tätigkeiten ihnen persönlich besonders liegen und welche ihnen aufgrund des Geschlechts zugewiesen werden. Bis auf wenige Ausnahmen sprachen die Männer jedoch auch davon, dass sie die erforderliche Arbeit im Team entlang der Maxime „alle machen alles“ zuteilen. Zugleich entstehen jedoch in den Alltagsroutinen auch Zuteilungen nach gender und maskuline Nischen, sodass trotz dieser Maxime beispielsweise handwerkliche Aufgaben und Fußball häufig den männlichen Kinderbetreuern zugewiesen werden.

In der von uns in diesem Praxisprojekt angebotenen Organisationsentwicklung wurde mit der Kitaleitung oder mit dem Kitateam die Aufgabenverteilung, die sich eingespielt hat, reflektiert. Drei Prinzipien wurden für Veränderungsprozesse thematisiert: Erstens sollen individuelle Stärken der Kinderbetreuenden in die Kita-Arbeit eingebracht werden können. Die Zuteilung nach gender führt auch dazu, dass stereotyp angefragt wird, ob jemand eine Tätigkeit übernehmen könnte, ohne nachzufragen, wer dafür individuell Kompetenzen mitbringt. Zweitens sollen diese individuellen Stärken so eingebracht werden, dass auch die anderen Kinderbetreuenden sich diese Kompetenzen aneignen. Dafür haben wir ein Buddy-System vorgeschlagen. Wenn eine Kinderbetreuerin beispielsweise besonders im Werken kompetent ist, soll auch eine weitere Kinderbetreuerin mit ihr zusammen dieses Angebot aufbauen und von ihr lernen. Drittens ist es wichtig, dass, wie die Kinderbetreuenden wiederholt ausgeführt haben, im Alltag tatsächlich ,alle alles machen“ was zum Betreuungs- und Bildungsauftrag der Kita gehört. Die Kinderbetreuenden sind aufgrund der Omnipräsenz von gender immer auch ein Rollenvorbild für die Kinder.

\subsubsection{Geschlechtergerechte Pädagogik: Interaktionen im Fokus}

Was aus den Ergebnissen des Forschungsprojekts folgt, unterstreicht nicht nur die Bedeutung des Tuns, sondern auch diejenige des Lassens. Wie im vierten Kapitel zu Interaktionen dargelegt, ist Dethematisierung als Reaktion von doing oder undoing gender durch das Kind die pädagogisch wertvollste Reaktion der Kinderbetreuenden. Ein Mädchen, dass Pink und Glitzer mag, soll dafür weder bestärkt noch kritisiert werden, genauso wie ein Junge, der die Fingernägel lackiert hat, darauf weder eine negative noch eine dramatisierend positive Reaktion benötigt. Dramatisierung von doing oder undoing gender, so die Schlussfolgerung, 
verstärkt Geschlechterstereotype. Durch die Dramatisierung wird das Kind entweder darauf hingewiesen, wie sehr es dem Geschlechterstereotyp entspricht, oder auch, wie sehr es sich in die Aktivitäten des anderen Geschlechts gewagt hat. Erfreulicherweise finden sich in der Analyse der Interaktionen wenig explizite Bestärkung von doing gender und keine abwertenden Reaktionen auf ein undoing gender der Kinder. Die Analyse zeigt jedoch auch, dass noch wenig Dethematisierung geschieht und dass die Geschlechterstereotypen als Erwartungsrahmen omnipräsent sind.

Um doing, undoing gender sowie Dramatisierung und Dethematisierung analytisch zu unterscheiden, wurden Videoanalysen auf der Mikroebene der Interaktionen realisiert. Die Diskussion der Beispiele aus dem Forschungsprojekt erwiesen sich in den Transferprojekten als sehr hilfreich. Mit Blick auf die Lehrmittel könnten die Beispiele und die Analyse für die Ausbildung ebenfalls genutzt werden. Interaktionen sind so fluide, dass es sich als schwierig erweisen kann, diese Praktiken zu verändern. Als statischer erweist sich der Raum. So sehr Kitas ihre räumlichen Arrangements den für sie relevanten historisch geprägten Pädagogiken anpassen, ebenso sehr drängt es sich auf, den heimlichen Lehrplan, das in den Artefakten und in der Raumordnung umgesetzte doing gender mit einem undoing gender im Raum herauszufordern. Wie ausgeführt erfordert ein undoing gender im Raum nicht nur die Sensibilität in Bezug auf beispielsweise die Diversität der vorhandenen Puppen, sondern auch die Beachtung der räumlichen Anordnung. Für eine geschlechtergerechte Pädagogik wird aufgrund der Forschungsergebnisse empfohlen, die Anordnung der Spielangebote gemäß der Arten des Spiels und der Themen vorzunehmen. Die Puppenstube wird so zum Straßenteppich gestellt und in Konsequenz können Praktiken des doing gender wie auch des undoing gender ermöglicht werden.

Insbesondere der analytische Blick unseres Forschungsprojekts auf Alltagspraktiken in der Kita ermöglichte eine konkrete Umsetzung der Erkenntnisse in den begleiteten Organisationsentwicklungsprozessen und Weiterbildungen. Die Omnipräsenz von gender in der Kita sowie die Möglichkeiten von doing und undoing gender, Dramatisierung und Dethematisierung wie auch das multiperspektivische Vorgehen in einer ethnografischen Forschungsstrategie kann, so hoffen wir, auch für andere Kontexte von Interesse sein. Dabei ist die methodische Verbindung von Interview, Beobachtung, Video und Dokumentenanalyse gut geeignet. Der Kontext der Kita ist ein besonders interessantes Feld: Kitas sind auch für die zukünftigen gesellschaftlichen Herausforderungen von großer Bedeutung; als Institutionen der frühen Bildung tragen sie zu Chancengleichheit bei; für die Vereinbarkeit und damit für die Gleichstellung der Geschlechter werden sie noch mehr an Bedeutung gewinnen. 


\section{Literatur}

Brandes, Holger, Andrä, Markus, Röseler, Wenke, \& Schneider-Andrich, Petra (2016). Macht das Geschlecht einen Unterschied? Ergebnisse der Tandem-Studie zu professionellem Erziehungsverhalten von Frauen und Männern. Opladen: Barbara Budrich.

Bruni, Attilla, Gherardi, Silvia, \& Poggio, Barbara (2005). Gender and Entrepreneurship. An Ethnographic Approach. London/New York: Routledge.

Connell, R. W. (2005). Hegemonic Masculinity: Rethinking the Concept. Gender \& Society, 19(6), 829-859.

Deutsch, Francine M. (2007). Undoing Gender. Gender \& Society, 21(1), 106-127.

Flick, Uwe. (2010). Qualitative Sozialforschung (3. Auflg.). Reinbek bei Hamburg: Rowohlt.

Gherardi, Silvia (1994). The Gender we Think, the Gender we Do in our Everyday Organizational Lives. Human Relations, 47(6), 591-610.

Gherardi, Silvia (1995). Gender, Symbolism and Organizational Cultures. London: Sage.

Goffman, Erving (1994). Das Arrangement der Geschlechter [The Arrangement Between the Sexes]. In Hubert A. Knoblauch (Hrsg. und Übersetz.), Interaktion und Geschlecht (S. 54-79). New York: Campus Verlag. (englisches Original 1977 erschienen).

Hirschauer, Stefan (2001). Ethnographisches Schreiben und die Schweigsamkeit des Sozialen. Zeitschrift für Soziologie, 30(6), 429-451.

Jeffrey, Bob, \& Troman, Geoff (2004). Time for Ethnography. British Educational Research Journal, 30(4), 535-548.

Kelle, Helga (2016). Herausforderungen ethnographischer Forschung zu Pädagogik und Geschlecht. In Ulrike Graff, Katja Kolodzig, \& Nikolas Johann (Hrsg.), Ethnographie - Pädagogik - Geschlecht: Projekte und Perspektiven aus der Kindheits- und Jugendforschung (S. 3-16). Wiesbaden: VS Verlag für Sozialwissenschaften.

Khan, Shamus R., \& Jerolmack, Colin (2013). Saying Meritocracy and Doing Privilege. The Sociological Quarterly, 54(1), 9-19.

Martin, Patricia Y. (2003). "Said and Done" versus "Saying and Doing”. Gendering Practices, Practicing Gender at Work. Gender \& Society, 17(3), 342-366.

Martin, Patricia Y. (2004). Gender as Social Institution. Social Forces, 82(4), 1249-1273.

Nentwich, Julia C. (2014). Puppen für die Buben und Autos für die Mädchen? Rhetorische Modernisierung in der Kinderkrippe. In Gerlinde Malli \& Susanne Sackl-Sharif (Hrsg.), Wider die Gleichheitsrhetorik. Soziologische Analysen-theoretische Interventionen. Texte für Angelika Wetterer (S. 50-61). Münster: Westfälisches Dampfboot.

Nentwich, Julia C., \& Kelan, Elisabeth K. (2014). Towards a Topology of 'Doing Gender': An Analysis of Empirical Research and its Challenges. Gender, Work \& Organization, 21(2), 121-134.

Schegloff, Emanuel A. (1997). Whose Text? Whose Context? Discourse \& Society, 8(2), $165-187$.

Schreier, Margrit (2010). Fallauswahl. In Günther Mey \& Katja Mruck (Hrsg.), Handbuch Qualitative Forschung in der Psychologie (S. 238-251). Wiesbaden: VS Verlag für Sozialwissenschaften.

Tennhoff, Wiebke, Nentwich, Julia C., \& Vogt, Franziska (2014). Gender in der Kita. Praxisratgeber für Kitaleitungen. St. Gallen: Universität St.Gallen und Pädagogische Hochschule St. Gallen. www.gender-kita.ch. 
Tennhoff, Wiebke, Vogt, Franziska, \& Nentwich, Julia C. (2017). Inklusion von Männern gemeinsam gestalten. Praxistipps für die Kita. St.Gallen: Universität St.Gallen und Pädagogische Hochschule St.Gallen. www.gender-kita.ch

Wetherell, Margaret (1998). Positioning and Interpretative Repertoires: Conversation Analysis and Post-Structuralism in Dialogue. Discourse \& Society, 9(3), 387-412.

Wetherell, Margaret, \& Edley, Nigel (2014). A Discursive Psychological Framework for Analyzing Men and Masculinities. Psychology of Men \& Masculinity, 15(4), 355-364.

Open Access Dieses Kapitel wird unter der Creative Commons Namensnennung 4.0 International Lizenz (http://creativecommons.org/licenses/by/4.0/deed.de) veröffentlicht, welche die Nutzung, Vervielfältigung, Bearbeitung, Verbreitung und Wiedergabe in jeglichem Medium und Format erlaubt, sofern Sie den/die ursprünglichen Autor(en) und die Quelle ordnungsgemäß nennen, einen Link zur Creative Commons Lizenz beifügen und angeben, ob Änderungen vorgenommen wurden.

Die in diesem Kapitel enthaltenen Bilder und sonstiges Drittmaterial unterliegen ebenfalls der genannten Creative Commons Lizenz, sofern sich aus der Abbildungslegende nichts anderes ergibt. Sofern das betreffende Material nicht unter der genannten Creative Commons Lizenz steht und die betreffende Handlung nicht nach gesetzlichen Vorschriften erlaubt ist, ist für die oben aufgeführten Weiterverwendungen des Materials die Einwilligung des jeweiligen Rechteinhabers einzuholen.

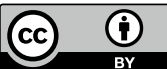

